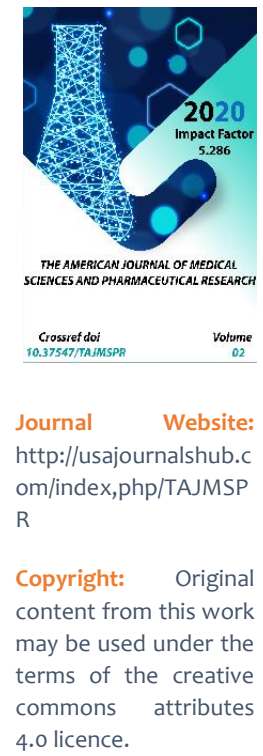

\title{
To Pathomorphology Of Idiopathic Fibrosing Alveolitis
}

Eshkabilov Tura Juraevich

Medical Candidate Of Sciences, Associate Professor Of Pathological Anatomy Department Of The Samarkand Medical Institute, Uzbekistan

Khamidova Farida Muinovna

Head Of The Department Of Pathological Anatomy Of The Samarkand Medical Institute, Candidate Of Medical Sciences, Associate Professor, Uzbekistan

Norjigitov Azamat Musakulovich

Assistant Of The Department Of Pathological Anatomy Of The Samarkand Medical Institute, Uzbekistan

\section{ABSTRACT}

Dysplasia is the most common form of morphological precancer of the cervix. Cytological smear from the cervical canal is one of the most effective methods for diagnosing cancer, background processes and precancerous conditions of the cervix at the very initial stages of occurrence. Histology allows you to determine the degree and form of epithelial damage, to detect the presence of atypical cells. The material for the study was targeted incisional biopsies from the cervix of 847 patients aged 19-73 years. All biopsies were stained with hematoxylin-eosin. Microscopic examination was performed using a conventional light microscope "Leica". The analysis of biopsy materials of 847 patients shows that $1 / 5$ of the patients $(20.8 \%)$ had dysplasia of varying severity. The highest peak of morbidity with this pathology occurs at the reproductive age, which makes this problem even more relevant for comprehensive study and discussion in a wide range of specialists. A comprehensive study of background and precancerous diseases is a great opportunity to detect the disease in time or prevent its occurrence. Therefore, every woman needs to undergo such a survey. 


\section{KEYWORDS}

Incisional biopsies, biopsies, dysplasia, precancerous cervix.

\section{INTRODUCTION}

The problem of nonspecific lung diseases in recent years all over the world, especially in industrially developed countries, is acquiring more and more practical and social significance due to the steady trend towards an increase in the incidence. In the United States, every fifth inhabitant suffers from one of the forms of nonspecific lung diseases, and the mortality rate from this pathology reaches 240 thousand annually $(1,2,3)$. In Uzbekistan, non-specific lung diseases rank third in the structure of morbidity, disability and mortality of the population $(1,2,6)$. Among chronic nonspecific diseases, fibrosing alveolitis constitutes a large group. The characteristic signs of fibrosing alveolitis about in are progressive shortness of breath, common sclerotic changes in the lungs and restriction syndrome. In MK B- 10, idiopathic fibrosing alveolitis (ELISA), exogenous allergic alveolitis (EAA) and toxic fibrosing alveolitis (TFA) are distinguished as independent nosological forms. Fibrosing alveolitis is a difficult to diagnose disease and its morphology does not have clear criteria. This is due to the multiple causes of this pathology and insufficient study of their morphodynamics. Although it has been more than 80 years, when it was first described the disease of American Pathologists J.Hamman and A.Rich, later named after their disease Hamman - Picha, there are still a lot of controversial issues in both the clinical and pathomorphological aspects of this disease. The aim of this study is to study the prevalence and pathomorphological changes of idiopathic fibrosing alveolitis on a sufficient autopsy material from the pathological department of the clinic of the Samarkand Medical Institute.

\section{MATERIAL AND RESEARCH METHODS}

For the analysis were taken all autopsy cases where clinical diagnosis fig chronic nonspecific pulmonary pathology. Total cases were analyzed for 30 years (1990-2020 years) was 268. The analysis of deaths from tuberculosis were excluded from pneumoconiosis and from sarcoidosis. The clinical diagnosis of these deaths are often appears chronic 
nonspecific pneumonia, chronic bronchitis, chronic astmatoidny bronchitis, congenital pulmonary anomaly, fibrosis and, chronic interstitial lung disease, chronic nonspecific pulmonary disease, idiopathic pulmonary lungs (fission syndrome - Gellerstedta), polycystic lungs and pneumoconiosis. The age of the deceased is 13-74 years. The greatest number of deaths were aged 37-61 years. 227 deaths ( $84,6 \%$ ) were male and only 41 (15,3\%) were female. It should be noted that only in 5 cases out of $268(1,8 \%)$ over the years mortem was exhibited disease Hamman - Picha. In all 268 autopsied cases, a thorough examination of the lung tissue was carried out. Sections were stained with hematoxylin - eosin, Van Gieson, Perls, Weigert and Donskov.

\section{RESEARCH RESULTS}

On the basis of thorough histological study targeted lung tissue were subjected to autopsy cases was found defeat interstitial lung tissue in the development of varying degrees of interstitial fibrosis in 17 deaths. Lung damage they wore progressively proliferative character and extended not only to interalvals partitions, but also on the interlobar structure, peribronchial and perivascular connective tissue. In some of those who died was observed pronounced peribronhiolit and obliterating the bronchial tubes of the litas.

Fragmentation of elastic fibers with edema of interstitial connective tissue and hyperplasia of argyrophilic fibers in the intervals septa, as well as metaplasiavery often, the transformation of flat and $\mathrm{X}$ alveolocytes into cubic epithelium was observed. In all 17 cases, multiple lymphoid, cell-recognized, plasmocytic, and Gist-and otsitar infiltrates of the interval septum were detected. In some of the deceased, the alveoli were filled with detached type II alveolites and macrophages. In a number of cases, small-cystic degeneration of the lung tissue was detected.

Damage to the vascular system of the lungs is very characteristic of all these 17 deceased. I observed in varying degrees of thickening of the vessel walls from narrowing their pros a veto, angiofibroz to form muftopodobnyh formations reflex-type vessels. At about all these observations were identified in varying degrees, expressed on I emphysema, especially strongly manifested in individuals with fine-cystic degeneration. Macroscopically, such lungs are identified as "honeycomb" lung. 
In the older deceased and with a long history, the lung parenchyma was severely destroyed and, due to pronounced fibrosis, the lung tissue was deformed, the alveolar epithelium resembled atypical tumor cells (but without mitotic figures and cell anaplasia), and microcalcifications were noted in sclerosed areas of the lung tissue.

\section{DISCUSSION AND ANALYSIS}

Thus, out of 268 deceased patients who were clinically diagnosed with chronic nonspecific lung disease (COPD), 17 cases revealed histological changes characteristic of progressive fibrosing alveolitis ( HammanPicha disease ). Interesting is the fact that unless the total number of deaths from the COPD (268) the largest part - 227 relates to a male and only 41 of women, of 17 cases, which were marked pathognomonic histological changes characteristic of the disease Hamman -Picha , 11 is female, accounting for $64,7 \%$ and only 6 is male.

On the Comparative often idiopathic fibrosing alveolitis women also noted CeglaU.H.(3,4,5,8), N.V. Putov and M.M. Ilkovich(6).
The autopsy material in 25 years only 5 dead mortem was put fibrosing alveolitis, and not in one of the 268 patients who died in the clinical diagnosis of the presence of the disease was not even suspected. This indicates a lack of awareness of doctors about as a stencil unjustified exposure of the diagnosis of chronic pneumonia in some chronic nonspecific diseases. Even the pathological diagnosis of Hamman's- Pich disease based on autopsy materials without a thorough histological examination sometimes does not clarify the essence of the true cause of death and the same COPD will appear in the statistics.

Pulmonologists and radiologists with a friendly and scrupulous examination of patients with progressive dyspnea, as a result of a careful differential diagnosis with common nosologies that manifest as COPD, can reveal this disease. For pathological verification of Hamman's - Pichadisease, it is necessary to exclude other nosologies and syndromes by the selection method and pay attention to pathognomonic structural changes in both lungs, which are characteristic of fibrosing alveolitis. 


\section{CONCLUSIONS}

Disease Hamman - Pich is a relatively rare, with poorly understood etiology, disease. Organopathology nature of the disease from progresses present character sclerosis deep lung and their irreversible changes. However, the wounds its recognition of this rare disease prolongs the life of patients.

There are appropriate word academician E.M. Tareeva(7). "Almost all diseases, including those that make up a kind of main diagnostic fund of the modern therapeutic clinic, were initially described as rare, so that after some period of" incubation ", which used to be calculated in decades, and now in years and even months, become very common."

\section{REFERENCES}

1. Abdullakhodjaeva M. S. Modern approaches to the study of pathological anatomy and pathogenesis of major human diseases. Assembly speech, Tashkent, 2007

2. Blinova S. A., Khamidova F. M., Ismoilov Zh. M. State of immune and regulatory structures of the bronchial mucosa in children with lung pathology. Journal of New days in medicine 29/1 2020 year:pages: 104-107. Bukhara.

3. Blinova S. A., Khamidova F. M., Ismoilov Zh. M. Dynamics of changes in the immune and endocrine structures of the lungs in experimental pneumonia. Biomedicine and practice. Magazine part 6, special issue no. SI-2 / 2020 page: 717721. Tashkent.

4. Blinova S. A., Khamidova F. M., Ismoilov Zh. M. Changes in the structural components of bronchial secretions in children with bronchiectatic disease. "Questions of science and education" No. 27 (76), 2019. Pages 16-24

5. Didkovsky N. A., Dvoretsky L. I. Hereditary factors and local protection in non-specific lung diseases. Moscow, 1990, p.4.

6. Putov N. V., Ilkovich M. M. Fibrosing alveolites, Leningrad, 1986, p. 35.

7. Tareev E. M. Rare diagnoses and rare diseases-therapeutic archive, 1974, no. 2, p. 3.

8. CeglaU.H. Die idiopatisch fibrosiendi Alveolitis. Die interstitiellen 
Doi: https://doi.org/10.37547/TAJMSPR/Volume02Issue09-03

Lungenfibrosen. - Jn: Bucherei oles

Pneumologen. Bd 2. Stuttgart, 1977, S. 1-

125. 